\title{
Imagerie musculaire et myopathies inflammatoires
}

\section{Françoise Chapon}

\section{Résumé}

Le diagnostic des myopathies inflammatoires idiopathiques (MII) s'appuie classiquement sur les données de la biopsie musculaire. Celle-ci permet de classer ces pathologies parmi quatre groupes existants: les dermatomyosites (DM), les myosites à inclusions (IBM), les myopathies nécrosantes autoimmunes (MNAI) et les myosites de chevauchement (dont le syndrome des antisynthétases ou ASyS).

L'apport des différentes techniques d'imagerie à visée diagnostique est exposé dans l'article cité en référence [1]. En résonance magnétique nucléaire (RMN), les séquences T2, STIR, et la méthode DIXON permettent d'apprécier l'œedème et les signes d'inflammation ; la séquence $\mathrm{T} 1$ révèle, quant à elle, l'involution graisseuse et l'atrophie du muscle. L'injection de gadolinium est inutile dans ce contexte. La biopsie musculaire reste plus sensible que la RMN pour démontrer une inflammation lorsque celle-ci ne met pas en évidence d'hypersignal. Pour autant, la RMN décrit une topographie des lésions différente selon les sous-types de MII (bien détaillée par les auteurs). La RMN musculaire ne s'avère pas utile dans l'aide au dépistage des complications graves des MII (pneumopathie interstitielle, néoplasies, myocardite), car le protocole d'acquisition des images est différent pour l'exploration des muscles et la recherche de ces complications. Les facteurs limitants de la RMN sont le caractère subjectif pour l'évaluation de l'hypersignal, et la non-spécificité des hypersignaux (observés notamment après exercice ou lors d'une dénervation). Dans le cadre du suivi des MII et l'évaluation des lésions musculaires, l'activité de la maladie musculaire est corrélée à l'existence d'hypersignaux en séquences T2. En cas de DM, l'évaluation de l'atrophie et de l'involution graisseuse, absentes initialement, se fait en séquence $\mathrm{T} 1$, nécessitant une comparaison avec les examens précédents ; en cas de MNAI et d'IBM, l'atrophie et l'involution adipeuse apparaissent précocement et sont plus importantes que dans les autres MII, mais avec une distribution différente. Pour le suivi, une RMN des cuisses (et non une RMN corps entier, cette dernière étant généralement réservée au diagnostic) est suffisante bien que la présence d'une atrophie et d'une involution adipeuse ne signe pas obligatoirement l'évolutivité de la maladie inflammatoire mais peut être liée à une autre étiologie.

Enfin, un grand chapitre est réservé au développement de l'imagerie musculaire, concernant l'amélioration de la RMN et d'autres méthodes d'imagerie.

\section{Commentaire}

Il s'agit d'une mise au point intéressante avec une bibliographie très exhaustive. L'utilité de la RMN dans l'étude des pathologies musculaires n'est plus à démontrer. Elle permet une aide au diagnostic étiologique, un guidage du geste biopsique (mais pas toujours), une quantification des lésions et un suivi évolutif de la pathologie. Les auteurs, après l'exposé des différentes techniques et leur apport spécifique dans l'analyse du tissu musculaire, discutent toutes ses indications notamment par rapport à la biopsie musculaire qui semble rester encore l'examen de référence pour la classification d'une MII. L'indication de la RMN la plus utile à l'heure actuelle dans le cadre du diagnostic étiologique des MII est la confirmation d'une IBM du fait de son profil caractéristique en RMN (sensibilité de $80 \%$ et spécificité de $100 \%)$. Elle apparait inutile pour le diagnostic dans le cas des DM du fait de la clinique très évocatrice. Dans les MNAI et ASyS, les anomalies manquent de spécificité. Pour le suivi des patients, il est important de retenir que l'examen peut se limiter à une exploration des cuisses et que la présence d'hypersignaux (en séquence T2) et l'involution adipeuse sont corrélées à l'atteinte clinique. Les limites de l'examen sont bien exposées et tiennent surtout à la subjectivité d'appréciation des hypersignaux et au manque de spécificité des anomalies tant pour le diagnostic que pour le suivi des pathologies. Enfin, l'encadré "BOX 1 " expose très clairement les recommandations proposées par les auteurs.

\section{Muscle imaging and inflammatory myopathies}

\section{LIENS D'INTÉRÊT}

L'auteure déclare n'avoir aucun lien d'intérêt concernant les données publiées dans cet article.
Françoise Chapon Centre de Compétence neuromusculaire, $\mathrm{CHU}$ de Caen, 14000 Caen,

France

Contact

francoise.chapon@ unicaen.fr

\section{RÉFÉRENCE}

1. Malartre S, Bachasson D, Mercy G, et al. MRI and muscle imaging for idiopathic inflammatory myopathies. Brain Pathol 2021 ; $31:$ e12954. 